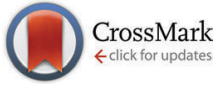

Cite this: J. Mater. Chem. C, 2015, 3, 10830

Received 6th August 2015, Accepted 18th September 2015

DOI: $10.1039 / c 5 t c 02446 c$

www.rsc.org/MaterialsC

\section{Efficiency increase of organic solar cells with emissive light-in-coupling layers $\uparrow$}

\author{
Beatrice Beyer*a and Karl Leo ${ }^{b}$ \\ Top-absorbing organic solar cells with a light incoupling layer allowing the exposure of harmful UV \\ irradiation have been fabricated. Short-wavelength light is absorbed by the down-shifting system \\ Alq $_{3}$ :DCM located in the light incoupling layer of top-absorbing organic solar cells and is converted into \\ longer wavelengths. This red light is then absorbed by a $\mathrm{ZnPc}: \mathrm{C}_{60}$ bulk heterojunction solar cell, \\ showing a total power efficiency increase by more than $10 \%$ related to additional photocurrent \\ generated by this architectural concept.
}

\section{Introduction}

Using photon conversion processes to positively influence the absorption behaviour is a common approach in the field of inorganic photovoltaics due to the discrete band structure of inorganic semiconductors. Photon conversion processes convert via luminescence parts of the solar spectrum to better match the absorption properties of a semiconductor device. 'Multi-photon' mechanisms such as up- and down-conversion, as well as single-photon processes like down-shifting can be used to achieve this goal. ${ }^{1,2}$

Here, the excellent fluorescent properties of 4-(dicyanomethylene)-2-methyl-6-( $p$-dimethylaminostyryl)-4H-pyran (DCM) will be exploited to study the concept of internal downshifting of the solar light for organic solar cells. The aim is to modify the absorption profile of binary bulk heterojunction (BHJ) solar cells with fullerene $\mathrm{C}_{60}$ as an electron acceptor and zinc phthalocyanine $(\mathrm{ZnPc})$ as an electron donor by utilizing the emission strength of DCM in the range between 580 and $780 \mathrm{~nm}$. DCM will be inserted into the light incoupling layer (LIL) of topabsorbing solar cells in order to absorb light and to convert it into lower energy radiation, as presented in Fig. 1.

Converting light of higher energy into lower energy by downshifting is accompanied by a loss of photon energy and will result in a non-negligible degree of heating of the semiconductors due to the Stokes shift. ${ }^{1}$ However, this heating has to be dealt with for

\footnotetext{
${ }^{a}$ Fraunhofer Institute for Organic Electronics, Electron Beam and Plasma Technology FEP, Maria-Reiche-Str. 02, 01109 Dresden, Germany. E-mail: beatrice.beyer@fep.fraunhofer.de

${ }^{b}$ Technische Universität Dresden, Institut für Angewandte Photophysik (IAPP), George-Bähr-Str. 1, 01069 Dresden, Germany

$\dagger$ Electronic supplementary information (ESI) available: Spectral overlap of $\mathrm{Alq}_{3}$ :DCM and $\mathrm{ZnPc}: \mathrm{C}_{60}$; results of optical simulations for various layers and thicknesses; optical constants of $\mathrm{Alq}_{3}$ and $\mathrm{Alq}_{3}: \mathrm{DCM}$. See DOI: 10.1039/c5tc02446c
}
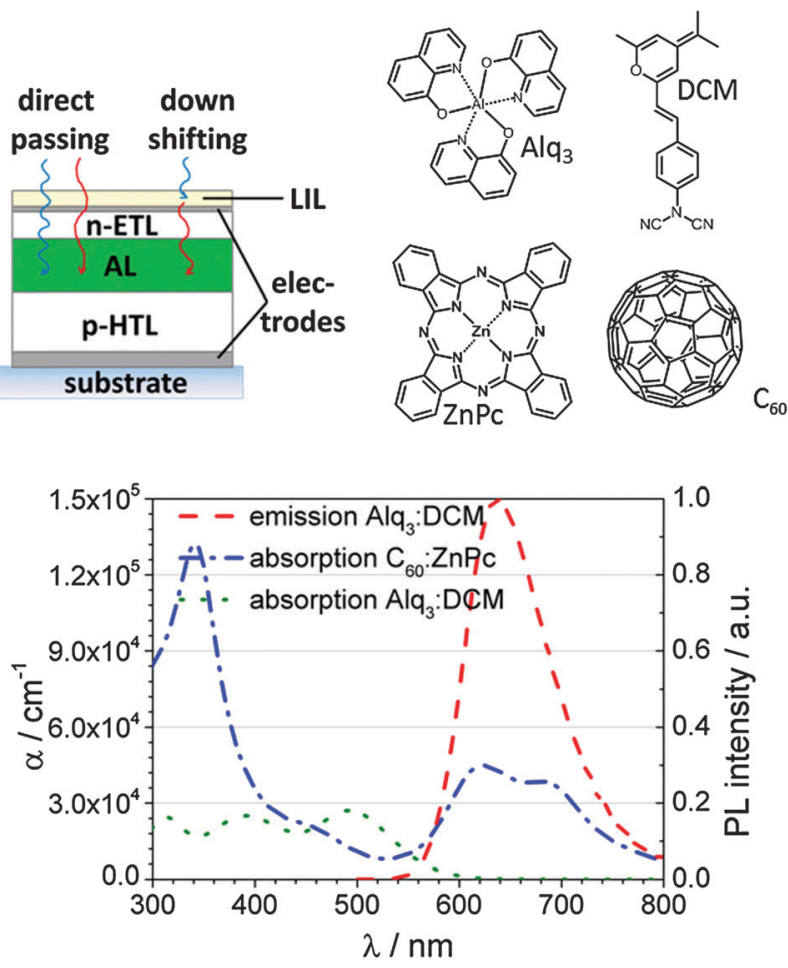

Fig. 1 Schematic presentation of the top-absorbing organic solar cell architecture (top left), the chemical structures of relevant compounds (top right) as well as their absorption and emission behaviour (bottom).

classical inorganic solar cells since the efficiency of these cells decreases at higher temperatures. This dependency of efficiency upon temperature is reversed for organic solar cells. ${ }^{3-5}$ So, the introduction of a down-shifting emitter molecule does not just convert the solar spectrum, it can also heat the cell from the inside. For our investigations the down-shifting emitter DCM is 
placed in the light incoupling layer conventionally consisting of pure tris(8-hydroxyquinolinato)aluminium $\left(\mathrm{Alq}_{3}\right)$ within the optoelectronic device without lowering the overall electrical performance of the bulk heterojunction (BHJ) organic solar cells (OSCs) by affecting also the fill factor (FF) or open circuit-voltage $\left(V_{\mathrm{OC}}\right)$, as often observed when sensitizers are added to the $\mathrm{BHJ}$ such as in ternary bulk heterojunction solar cells. ${ }^{6,7}$

\section{Experimental}

\section{Sample preparation}

Solar cells were fabricated by a consecutive (co-)evaporation of organic materials in a custom-made organic material beam deposition tool (Bestec, Germany) at a pressure of $10^{-8}-10^{-7} \mathrm{mbar}$ and at room temperature. A pre-cleaned bare glass (Luminescence Technologies) was used as a substrate. We used a $\mathrm{p}-\mathrm{i}-\mathrm{n}$ device architecture in order to provide ohmic contacts to both electrodes. ${ }^{8}$ The active layer $\left(\mathrm{ZnPc}: \mathrm{C}_{60}\right.$, materials were twice sublimed prior to utilization) was embedded between an anode consisting of $50 \mathrm{~nm}$ aluminum and $30 \mathrm{~nm}$ silver, a p-doped hole transport layer (HTL) of $2,2^{\prime}, 7,7^{\prime}$-tetra( $m$-tolyl-phenylamino)-9,9'-spiro-bifluorene (spiroTTB) in varied thicknesses and a $20 \mathrm{~nm}$ thick blocking layer of $\mathrm{C}_{60}$ (BL), followed by an n-doped electron transporting layer of $15 \mathrm{~nm} \mathrm{C_{60 }}$ (ETL) and a cathode of $3 \mathrm{~nm}$ aluminium and $12 \mathrm{~nm}$ silver. For processing reasons, $\mathrm{p}$ - and $\mathrm{n}$-doping was done employing the proprietary molecular dopant NDP9 and NDN26 (Novaled AG, Germany), which could be replaced by the open materials 2,3,5,6-tetrafluoro-7,7,8,8-tetracyanoquino-dimethane (F4-TCNQ) and $\mathrm{Cs}_{2} \mathrm{CO}_{3}$, respectively. For the specific investigations (HTL thickness variation, DCM dopant concentration variation) all samples and layers were fabricated within one single deposition run by either applying a wedge tool (for HTL thickness increase without interrupting the process) or specially structured shadow masks (for doping variation). Each OSC sample obtained four pixels with an active area of $4.5 \times 5(\mathrm{~mm})^{2}$ for characterisation to investigate the statistical deviation.

\section{Sample characterisation}

$J-V$ measurements (Keithley 2400 source meter, Kepco power supply) are recorded under dark conditions and under AM1.5 and $100 \mathrm{~mW} \mathrm{~cm}{ }^{-2}$ illumination (KHS Technical lighting) at $25^{\circ} \mathrm{C}$. The optical constants were determined by transmission and reflection spectroscopy (Solid Spec, Shimadzu) and by subsequent data fitting with the software WVASE32 (J.A.Woollam Co., Inc.). Spectral response curve data were recorded using a spectrofluorometer (Horiba Jobin Yvon, Fluoromax-4, also used for photoluminescence measurements) calibrated with a Si photodiode and a Keithley 2400 source meter. Along with the short current density $\left(J_{\mathrm{SC}}\right)$, the fill factor $(\mathrm{FF})$, open circuit voltage $\left(V_{\mathrm{OC}}\right)$, and power efficiency $(\eta)$, the saturation $(S)$ is given, defined as the current density ratio at $-1 \mathrm{~V}$ and $0 \mathrm{~V}\left(S=J(-1 \mathrm{~V}) / J_{\mathrm{SC}}\right)$.

\section{Optical simulations}

In order to investigate the field distribution in optoelectronic devices, the simulation software Setfos 3.2 (Fluxim, Switzerland) was used. Optical constants were determined by transmission and reflection spectroscopy. The field amplitudes and the energy fluxes for each wavelength, at each position within the stack were calculated by using a transfer matrix formalism implementing the optical constants of each layer. For optimization of the transport layer thicknesses, the absorptivity of the absorption layer was maximized for the AM1.5 solar spectrum.

\section{Results and discussion}

\section{Optical properties of $\mathrm{Alq}_{3}: \mathrm{DCM}$}

DCM has widely been used as an emitter dopant material with $\mathrm{Alq}_{3}$ as a host system in organic light emitting diodes (OLEDs). Remarkably, DCM shows a distinct solid solvation dependency in $\mathrm{Alq}_{3} .{ }^{9}$ This effect is expressed in the form of a concentration dependent emission colour and intensity. The more emitter is doped into $\mathrm{Alq}_{3}$, the more the emission is bathochromically shifted. This has been ascribed to the self-polarization of the DCM molecule, since it is more polar than the average organic semiconductor material or $\mathrm{Alq}_{3}$, respectively. Because of this dependence of emission upon concentration, thin films with different DCM concentrations were fabricated and characterized. Fig. 2a shows the transmission spectra of various $\mathrm{Alq}_{3}: \mathrm{DCM}$ thin films with different compositional ratios. The more DCM is added, the stronger the transmission at around $495 \mathrm{~nm}$ is reduced; this is due to the increasing absorption of DCM. Fig. 2b shows the PL spectra of these layers excited at $495 \mathrm{~nm}$ for various DCM contents. As mentioned before, the emission shows a bathochromic shift due to the increasing polarity with rising DCM content. After reaching a doping concentration of $10 \mathrm{wt} \%$, the emission is not further red-shifted. Additionally, the emission originating from $\mathrm{Alq}_{3}$ is immediately reduced after the addition of DCM. This is a clear sign of an efficient Förster resonance energy transfer (FRET). In order to investigate the excitation and emission characteristics of pure $\mathrm{Alq}_{3}$ and DCM-doped $\mathrm{Alq}_{3}$, emission spectra have been recorded for excitation wavelengths between 270 and $480 \mathrm{~nm}$. These 3D spectra are presented in Fig. $2 \mathrm{c}$ and $\mathrm{d}$ for pure and doped $\mathrm{Alq}_{3}$, respectively. In both graphs the emission has been measured from 500 to $800 \mathrm{~nm}$, where it matches with the $\mathrm{C}_{60}: \mathrm{ZnPc}$ absorption of $\mathrm{BHJ}$ OSCs. The spectrum of pure $\mathrm{Alq}_{3}$ is characterized by green emission which exhibits its lowest energy excitation maximum at $370 \mathrm{~nm}$. It can be seen that the wavelength of the emission maximum increases with rising excitation wavelength, whereas it stays almost unchanged in the doped $\mathrm{Alq}_{3}$ thin film. The emission in this case is characterized by a red colour with high intensity. The existence of FRET is attested to the unchanged location of the lowest energy excitation maximum at around $368 \mathrm{~nm}$, which means that the energy absorbed by $\mathrm{Alq}_{3}$ is directly transferred to the energy acceptor molecule DCM. Beyond the excitation of $\mathrm{Alq}_{3}$, DCM still emits by itself with a slightly lower intensity. Thus, red emission can be realized by excitation between 270 and $550 \mathrm{~nm}$.

Light incoupling layers are used to influence the optical field distribution and its amplitude, and to reduce the external reflection of the semitransparent top contact. Whereas a strong 

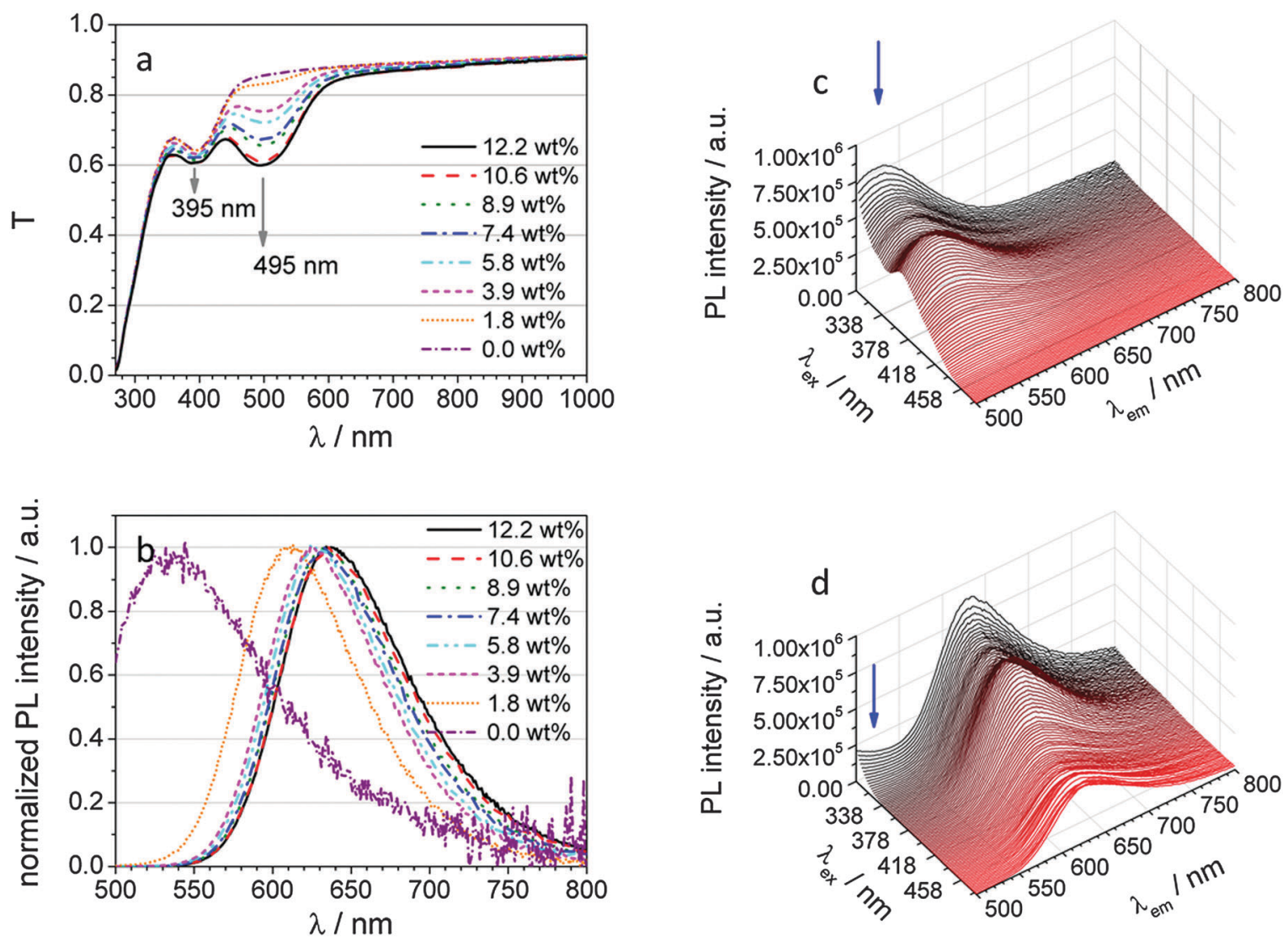

Fig. 2 Transmission (a) and normalized PL spectra (b) of $80 \mathrm{~nm}$ Alq 3 :DCM thin films depending on the DCM concentration as well as 3D photoluminescence spectra of thin films of pure $\mathrm{Alq}_{3}$ (c) and $\mathrm{Alq}_{3}$ doped with 10 wt\% DCM (d).

correlation between the LIL and the $J_{\mathrm{SC}}$ exists, Meiss et al. described minor influences on the $V_{\mathrm{OC}}, \mathrm{FF}$, and saturation values. ${ }^{10}$ Because of the convenient possibility of tuning the emission colour by varying the doping concentration, this LIL can be adjusted for several electron donor systems of the active solar cell layer absorbing between 500 and $700 \mathrm{~nm}$. Furthermore, with the combination of DCM and $\mathrm{Alq}_{3}$, another advantage arises. Pure $\mathrm{Alq}_{3}$ has a large absorption coefficient $\left(\alpha>10^{5} \mathrm{~cm}^{-1}\right.$ from 250 to $300 \mathrm{~nm}, \alpha>10^{4} \mathrm{~cm}^{-1}$ from 300 to $420 \mathrm{~nm}$ ) in the UV to blue range while staying below $10^{-1} \mathrm{~cm}^{-1}$ for longer wavelengths. This means that more than a half of the near-UV light is absorbed by a film of only $40 \mathrm{~nm}$ thickness.

Additionally, pure $\mathrm{Alq}_{3}$ shows a green emission, whose intensity is, however, still two magnitudes lower than the doped film, thus rather weak. Due to the strong absorption between 300 and $440 \mathrm{~nm}$, the energy rich UV/vis radiance, often attributed to the degradation of several organic semiconductors in organic solar cells, is filtered out before the organic solar cell. ${ }^{11}$ Adding $\mathrm{DCM}$ into $\mathrm{Alq}_{3}$ broadens the absorption range of energy rich radiance to $550 \mathrm{~nm}$, although it is not anticipated for this experiment. Additionally, the energy is not lost for the solar cell as it would be the case for pure $\mathrm{Alq}_{3}$. The absorbed light with a wavelength as short as $400 \mathrm{~nm}$ will be transferred from $\mathrm{Alq}_{3}$ to DCM via FRET, resulting in radiative decay as red emission. This can easily be absorbed by the $\mathrm{C}_{60}: \mathrm{ZnPc}$ containing absorption layer of OSCs, as shown by the spectral overlap of $\mathrm{Alq}_{3}: \mathrm{DCM}$ emission with $\mathrm{C}_{60}: \mathrm{ZnPc}$ absorption. The normalized spectra (cf. ESI, $\dagger$ S1) have an overlap of $99.5 \%$.

\section{Optical simulations}

In order to apply a fluorescent LIL in organic solar cells, a stack architecture for top-absorbing solar cells is utilized. The principle architecture has been adopted from ref. 10 but has been modified. Several optical simulations have been carried out in order to locate the electric field amplitude maximum in the absorption layer as it is of great interest for solar cells in top-absorbing geometry. ${ }^{12,13}$ The optical simulations started with a fixed stack architecture, and the variation was carried out for each layer. The results are presented in the supplementary information for pure $\mathrm{Alq}_{3}(\mathrm{ESI}, \dagger \mathrm{S} 2)$ and doped $\mathrm{Alq}_{3}(\mathrm{ESI}, \uparrow \mathrm{S} 3)$ as a LIL material. The absorptivity of $\mathrm{C}_{60}: \mathrm{ZnPc}$ shows a dependency upon all layers in the spectral region from 550 to $750 \mathrm{~nm}$, however with different sensitivities. Whereas the thicknesses of the BL, ETL and HTL have an enormous impact on the position of the optical cavity, the influence of the LIL is comparatively low. For clarification, the predicted $J_{\mathrm{SC}}$ under the assumption of AM1.5 illumination is illustrated, too. It has to be noted that the impact of emissive layers could not be considered in these simulations.

Due to the parasitic absorption of the BL and ETL, their thicknesses were limited for simulations revealing only the first 
field amplitude maximum. However, because of processing reasons, the second thickness maxima were not considered for the HTL and LIL. Furthermore, it can be seen that a thin semitransparent cathode is essential for high $J_{\text {SC }}$ values. Hence, a thickness for an optimal compromise between high transparency and good conductivity should be chosen.

The simulations for pure and doped $\mathrm{Alq}_{3}$ as light incoupling layers show that the dopant (concentration) has only a minor influence on the generated photocurrent, although the optical behaviour of the LILs is rather different ( $c f$. S4 and S5, ESI $\dagger$ ).

Because of the good agreement between the pre-assumed stack architecture and the simulation results, only the thickness of the HTL was experimentally varied for optimization purposes. Hence, an organic solar cell consisting of the following layer

Table 1 Key values of top-absorbing organic solar cells depending on their HTL thickness

\begin{tabular}{llllll}
\hline$d_{\mathrm{HTL}} / \mathrm{nm}$ & $V_{\mathrm{OC}} / \mathrm{mV}$ & $J_{\mathrm{SC}} / \mathrm{mA} \mathrm{cm}^{-2}$ & $\mathrm{FF} / \%$ & $\eta / \%$ & $S$ \\
\hline 45 & $503 \pm 9$ & $4.23 \pm 0.07$ & $48 \pm 0.5$ & $1.03 \pm 0.02$ & $1.18 \pm 0.02$ \\
55 & $508 \pm 13$ & $5.20 \pm 0.08$ & $49 \pm 0.5$ & $1.31 \pm 0.03$ & $1.21 \pm 0.02$ \\
60 & $506 \pm 12$ & $5.40 \pm 0.07$ & $49 \pm 0.5$ & $1.35 \pm 0.02$ & $1.15 \pm 0.02$ \\
65 & $536 \pm 14$ & $5.63 \pm 0.07$ & $45 \pm 0.4$ & $1.36 \pm 0.02$ & $1.15 \pm 0.02$ \\
70 & $516 \pm 11$ & $5.61 \pm 0.08$ & $46 \pm 0.5$ & $1.36 \pm 0.03$ & $1.17 \pm 0.02$ \\
80 & $538 \pm 14$ & $5.58 \pm 0.06$ & $47 \pm 0.5$ & $1.42 \pm 0.02$ & $1.17 \pm 0.02$ \\
90 & $519 \pm 8$ & $4.84 \pm 0.07$ & $43 \pm 0.4$ & $1.10 \pm 0.02$ & $1.21 \pm 0.02$
\end{tabular}

sequence will be investigated: a glass/opaque anode of aluminium and silver/ $x \mathrm{~nm}$ p-spiro-TTB, a $35 \mathrm{~nm} \mathrm{AL} / 20 \mathrm{~nm} \mathrm{BL} / 15 \mathrm{~nm}$ ETL/15 nm cathode of aluminium and silver/80 nm LIL. Since the emissive LIL and the absorbing AL are separated by around $50 \mathrm{~nm}$, which is too large for FRET, the magnitude of the incoupling will be investigated by a variation of the DCM concentration in the LIL to directly study the effect of this approach.

\section{Variations in the HTL thickness}

As summarized in the ESI, $\dagger$ (S2, S3), the dependency of high photocurrents on thickness is most pronounced for the HTL. A continuous increase of the AL thickness has led to a significant drop in FF and was kept at 35 or $40 \mathrm{~nm}$. Thus, samples with seven different HTL thicknesses ranging from 45 to $90 \mathrm{~nm}$ were fabricated in order to investigate the best thickness to enable high $J_{\mathrm{SC}}$ values. Table 1 gives an overview of the resulting key values of the devices. As expected, FF and $V_{\mathrm{OC}}$ show no significant dependency on the HTL thickness. This is attributed to the high conductivity of the p-HTL, preventing charge carrier losses due to high resistance. However, the scattering of the values is higher than expected from the evaluated measurement uncertainty. Nonetheless, a clear relation between $J_{\mathrm{SC}}$ and the HTL thickness can be drawn. Fig. $3 \mathrm{a}$ and $\mathrm{b}$ present the measured $J-V$ curves in the dark and under illumination and
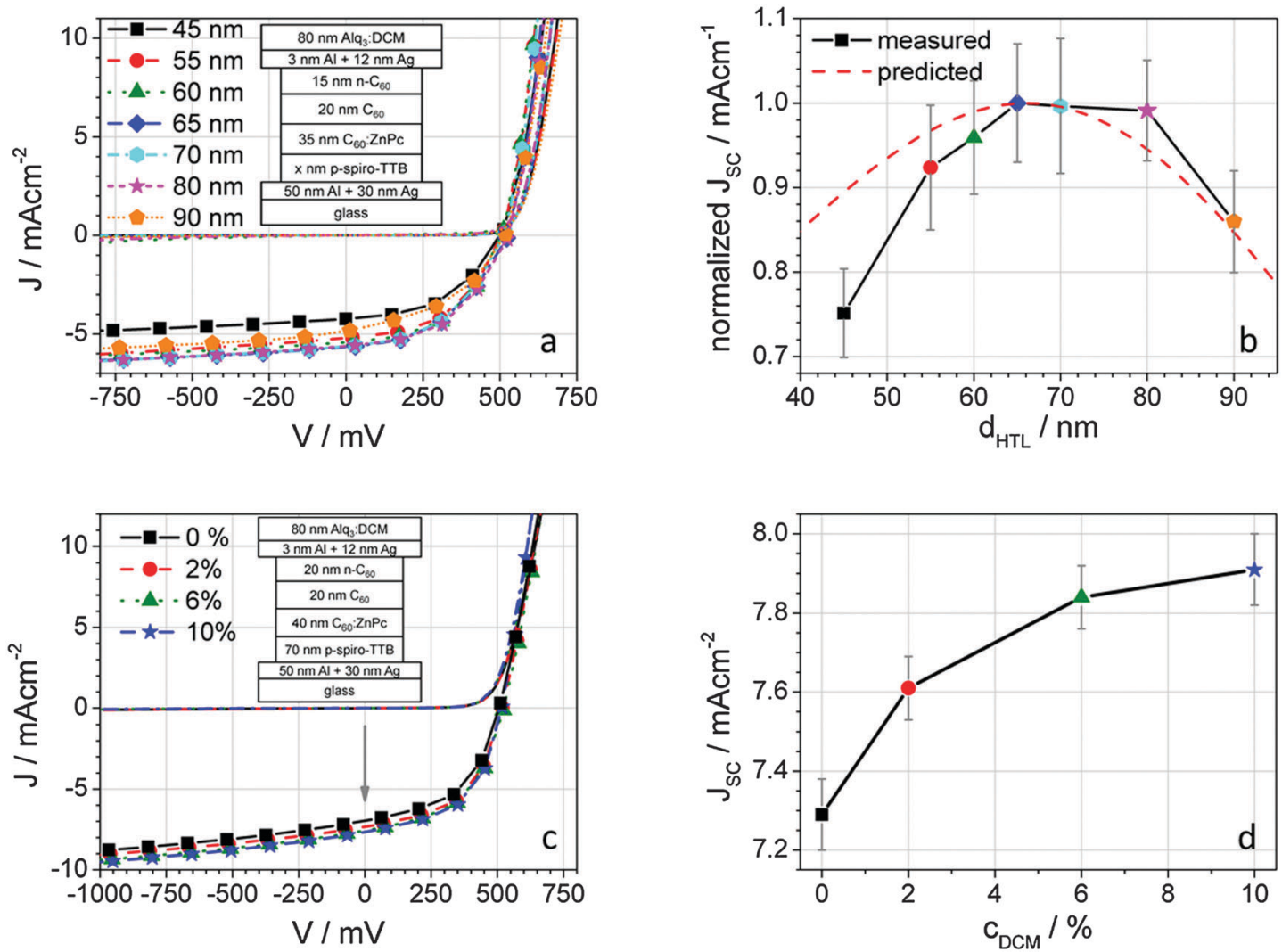

Fig. $3 \mathrm{~J}-\mathrm{V}$ characteristics of top-absorbing OSCs under illumination (line + symbol) and in the dark (line) depending on the hole transport layer (HTL) thickness (a) and concentration of DCM doped in $\mathrm{Alq}_{3}$ (c) as well as their $\mathrm{J}_{\mathrm{SC}}$ values plotted against the thickness (b) in comparison with the predicted values from simulations and against the DCM concentration (d). 
shows the evaluated $d_{\mathrm{HTL}}-J_{\mathrm{SC}}$ relation in comparison with the predicted $J_{\mathrm{SC}}$ values from the optical simulations. Clearly, $J_{\mathrm{SC}}$ has a maximum for thicknesses in the range between 65 and $80 \mathrm{~nm}$ and the results are consistent with the predicted behaviour. According to the simulations, a maximum short circuit current density is reached for an HTL thickness of $65 \mathrm{~nm}$. The experiments show that slightly higher thicknesses also give very good photocurrents. This reproduction shows that the optical model used as well as the applied parameter sets of the optical constants are in good agreement with reality.

\section{Impact of the DCM concentration within the LIL}

In order to validate the justification of the DCM insertion into the LIL, the DCM concentration was varied from 0 to $10 \mathrm{wt} \%$. The $J-V$ curves recorded under illumination and in the dark of the resulting OSC ( $c f$. Fig. $3 c$ and d) show samples with high fill factors and $V_{\mathrm{OC}}$ values as expected for $\mathrm{C}_{60}: \mathrm{ZnPc} \mathrm{BHJ}$ cells. The key values of the resulting devices are summarized in Table 2 . Since the LIL is not involved electrically in the photovoltaic processes, parameters such as the $\mathrm{FF}, V_{\mathrm{OC}}$, and $S$ are not significantly affected by the dopant concentration. Merely the short-circuit current density $J_{\mathrm{SC}}$ increases with rising DCM content in the LIL. Starting with the top-absorbing sample with pure $\mathrm{Alq}_{3}$ as a LIL material, short circuit current densities of $7.20 \mathrm{~mA} \mathrm{~cm}{ }^{-2}$ are obtained yielding an efficiency of $1.90 \%$, which is slightly higher than reported by Meiss and co-workers for a comparable architecture (1.84\%). ${ }^{10}$ After adding DCM to $\mathrm{Alq}_{3}$ by co-deposition, the emission intensity of the LIL increased and changed from greenish to orange-reddish; the BHJ absorption layer can better absorb the red light re-emitted by the LIL. This is confirmed by the EQE measurements, which will be discussed later in detail. Interestingly, the enhancement of the photosensitivity saturates quickly, as displayed in Fig. 4 b. The highest jump of $0.41 \mathrm{~mA} \mathrm{~cm}^{-2}$ is observed at $2 \%$ DCM addition. Addition of further DCM to a dopant concentration of $6 \%$ enhances $J_{\mathrm{SC}}$ by $0.21 \mathrm{~mA} \mathrm{~cm}^{-2}$, and after another $4 \% \mathrm{DCM}$ (a total content of $10 \%$ ) only by $0.12 \mathrm{~mA} \mathrm{~cm}^{-2}$.

In order to analyse these results further, reflectance and emission spectra have been recorded for these samples. By means of the reflectance spectra, the absorption of the whole device can be evaluated. As displayed in Fig. 4a, the absorption peaks originating from $\mathrm{Alq}_{3}(405 \mathrm{~nm})$, DCM (505 nm) and ZnPc (625$690 \mathrm{~nm}$ ) can be assigned. Whereas the absorption stays nearly unchanged for $\mathrm{Alq}_{3}$ and ZnPc related bands, the DCM band increases noticeably with rising dopant concentration, which results, for the sample with 10\% DCM in the LIL, to an absorption value comparable to that of the $\mathrm{ZnPc}$ band. These measurements

Table 2 Key values of top-absorbing organic solar cells depending on the DCM concentration in the light incoupling layer

\begin{tabular}{llllll}
\hline$C_{\mathrm{DCM}} / \%$ & $V_{\mathrm{OC}} / \mathrm{mV}$ & $J_{\mathrm{SC}} / \mathrm{mA} \mathrm{cm}^{-2}$ & $\mathrm{FF} / \%$ & $\eta / \%$ & $S$ \\
\hline 0 & $514 \pm 7$ & $7.20 \pm 0.09$ & $51 \pm 0.5$ & $1.90 \pm 0.02$ & $1.21 \pm 0.02$ \\
2 & $524 \pm 7$ & $7.61 \pm 0.08$ & $53 \pm 0.4$ & $2.11 \pm 0.02$ & $1.19 \pm 0.02$ \\
6 & $537 \pm 8$ & $7.80 \pm 0.08$ & $52 \pm 0.4$ & $2.16 \pm 0.03$ & $1.19 \pm 0.02$ \\
10 & $525 \pm 6$ & $7.92 \pm 0.09$ & $53 \pm 0.4$ & $2.21 \pm 0.02$ & $1.19 \pm 0.02$
\end{tabular}
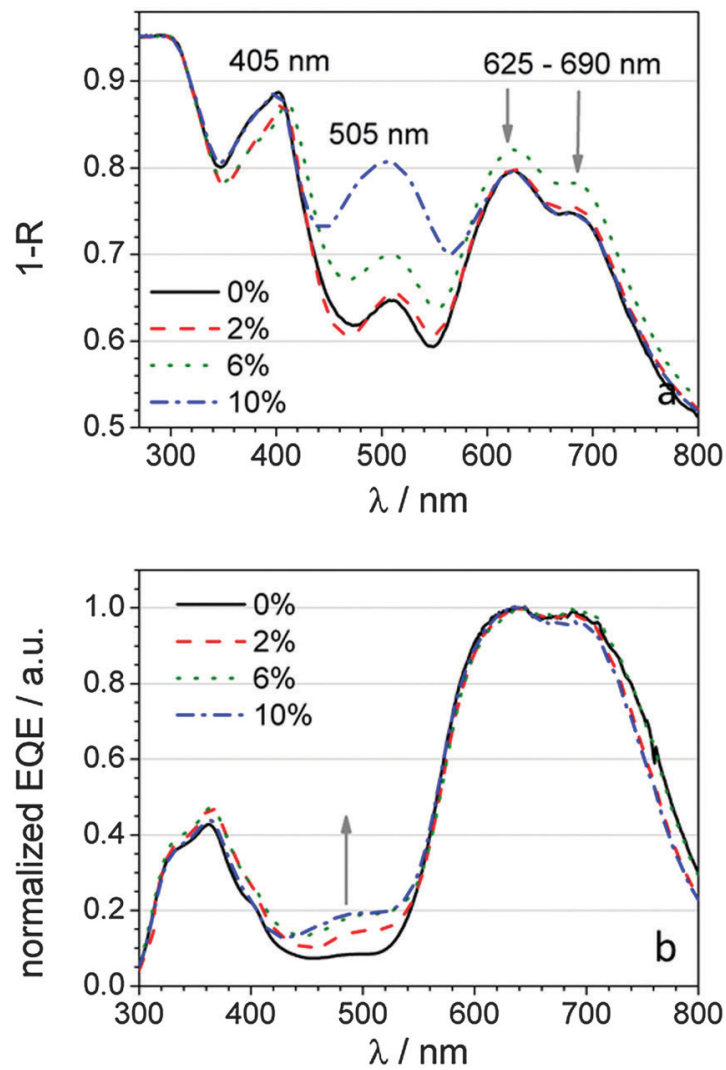

Fig. 4 (a) Absorption spectra of the devices as well as (b) their corresponding normalized EQE measurements depending on the DCM concentration in the light incoupling layer.

show that the absorption profile of the devices can be considerably altered by the choice of the LIL composition.

However, there is often a huge difference between the absorption capability and the generation of free charge carriers. Unlike the absorption spectra, the EQE spectra show the dominant $\mathrm{Q} / \mathrm{Q}+$ bands of $\mathrm{ZnPc}$ and the superposition of $\mathrm{C}_{60}$ and $\mathrm{Alq}_{3}$ absorption. For clarification, the spectra are normalized at the ZnPc related bands because all the layers beyond the LIL have been kept equal. At $480 \mathrm{~nm}$ an absorption shoulder rises and increases with DCM content. For the interpretation of the EQE spectra, a discussion of the emission properties of the light incoupling layers is required.

The PL spectra of all solar cell samples are presented in Fig. 5 and show the emission spectra of all four samples at various excitation wavelengths. These wavelengths have been selected to correspond to shoulders or maxima in the EQE spectra. Depending on the excitation wavelength, transitions occurring in either $\mathrm{Alq}_{3}$ (330, 365 and $405 \mathrm{~nm}$ ) or DCM $(480 \mathrm{~nm})$ are excited. The device with pure $\mathrm{Alq}_{3}$ has a yellowish emission with rising intensity for longer excitation wavelengths. Emission in the red region is not observed. After inserting 2\% DCM into $\mathrm{Alq}_{3}$, all the excitation energy is transferred to the DCM molecule via FRET, resulting in red emission. Furthermore, the DCM molecule can also be directly excited by illuminating it with $480 \mathrm{~nm}$ light. Further addition of DCM to 6\% has increased the emission intensity and shifts it slightly bathochromically. 

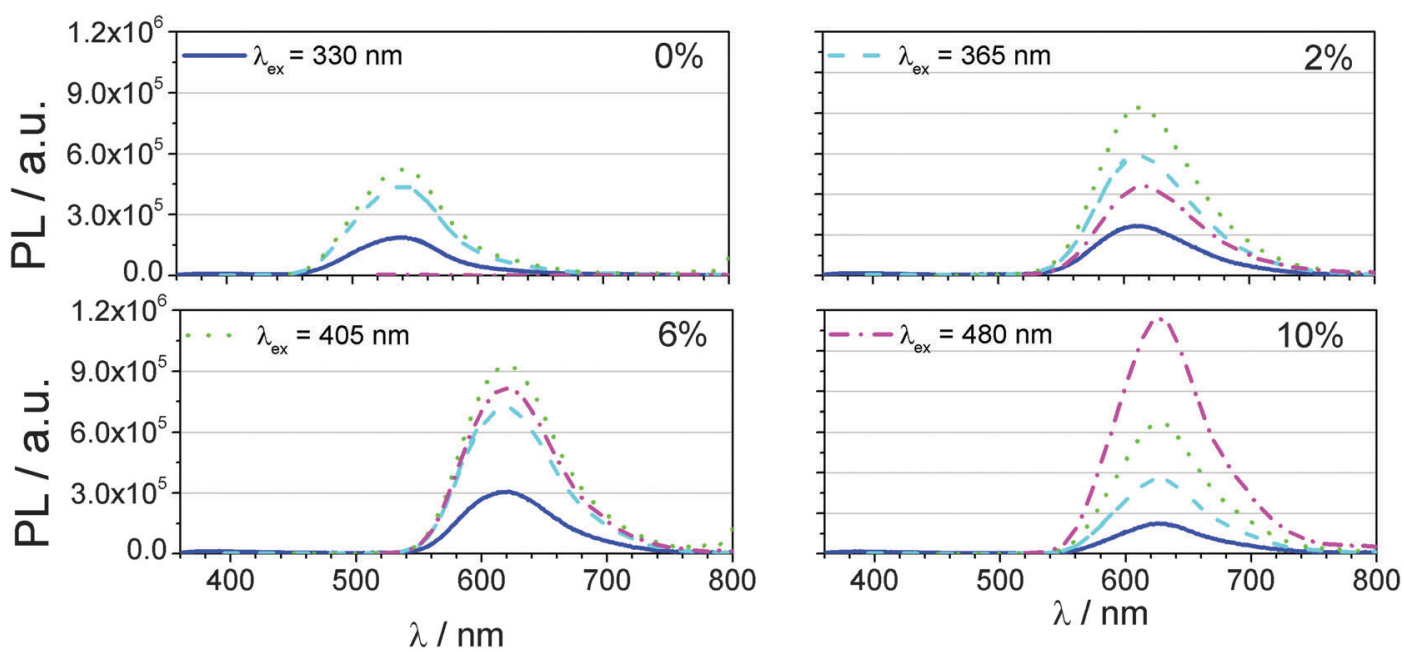

Fig. $5 \mathrm{PL}$ spectra of top-absorbing OSCs with different LILs in variation of their DCM doping concentration (0-10\%). These spectra are depicted for the excitation wavelengths at $330 \mathrm{~nm}$ (line), $365 \mathrm{~nm}$ (dashed), $405 \mathrm{~nm}$ (dotted) and $480 \mathrm{~nm}$ (dash-dotted).

Beyond that, the direct excitation of DCM becomes more effective. This observation continues to be true up to a content to $10 \%$. The highest intensity of red light is achieved after direct excitation of DCM, for which the energy transfer from $\mathrm{Alq}_{3}$ is less efficient compared to lower DCM concentrations.

A comparison between these results and the EQE spectra (Fig. 4b) explains the steep increase of the short circuit current density after the insertion of $2 \%$ DCM into the LIL and the subsequent saturation. The emission from pure $\mathrm{Alq}_{3}$ is green and can hardly be absorbed by ZnPc. Due to the effective FRET from $\mathrm{Alq}_{3}$ to DCM, photon energy absorbed by DCM and most notably by $\mathrm{Alq}_{3}$ can be utilized for exciton generation in the $\mathrm{C}_{60}: \mathrm{ZnPc}$ blend because of the matching spectral overlap between the red DCM related emission and the absorption profile of ZnPc. Up to 6\% of DCM, the effectivity of this FRET and the emission intensity increase with doping concentration. This can be seen in the EQE spectra with a higher sensitivity at $360 \mathrm{~nm}\left(\mathrm{Alq}_{3}\right.$ excitation) and $480 \mathrm{~nm}$ (DCM excitation). At 10\% DCM content, the sensitivity at $360 \mathrm{~nm}$ drops back to a value prior to DCM doping and reveals only a higher response at $480 \mathrm{~nm}$, which means that mainly direct excitation of DCM occurs. Hence, higher DCM concentrations in the LIL will not increase the $J_{\mathrm{SC}}$ value further. The solar energy irradiated from 300 to $420 \mathrm{~nm}$ yields only $71 \mathrm{~mW} \mathrm{~m}^{-2} \mathrm{~nm}^{-1}$ and increases up to $247 \mathrm{~mW} \mathrm{~m}^{-2} \mathrm{~nm}^{-1}$ within the spectral range of 300 to $540 \mathrm{~nm}$, which is around the half of the spectral range from 300 to $800 \mathrm{~nm}$ with $589 \mathrm{~mW} \mathrm{~m}^{-2} \mathrm{~nm}^{-1}$. Thus, enhancing the absorptivity in the blue range of light can only account for $12 \%$ of the achievable energy from 300 to $800 \mathrm{~nm}$. Addressing also the part of the spectrum till $540 \mathrm{~nm}$, already $42 \%$ of the irradiated energy is available for charge generation. Keeping this in mind, the $J_{\mathrm{SC}}$ improvement resulting from the addition of $2 \%$ is with $0.4 \mathrm{~mA} \mathrm{~cm} \mathrm{~cm}^{-2}(5 \%)$ remarkable and maximizes at $10 \mathrm{wt} \%$ by $0.72 \mathrm{~mA} \mathrm{~cm}{ }^{-2}(10 \%)$, although becomes less efficient due to direct absorption of DCM.

However, since the doped LIL has the main purpose of accessing filtered UV light $(\sim 300$ to $400 \mathrm{~nm})$, the absorption increase at around $500 \mathrm{~nm}$ can be considered as parasitic since broader absorbing solar cell systems would negatively be influenced and result in lower power conversion efficiencies. From this aspect, the sample with only $2 \% \mathrm{DCM}$ in $\mathrm{Alq}_{3}$, but predominantly only contributing by $\mathrm{Alq}_{3}$ absorption represents the best choice.

In order to increase the positive contribution of the emissive LIL further, a special layer design could be applied where less than $50 \%$ of the LIL emission is directed out of the solar cell into the air. This could be realized by an anisotropic orientation of the dopant emitter or increasing the reflectivity of the LIL/air interface.

\section{Conclusions}

We present organic solar cells which harvest the higher energy part of sun light more effectively by applying an emissive light incoupling layer on a top-absorbing cell structure. Due to the efficient down-shifting properties of $\mathrm{Alq}_{3}: \mathrm{DCM}$, the overall power efficiency has been increased by 10\% (with 2\% DCM in $\mathrm{Alq}_{3}$ ) and demonstrates that emission can effectively be absorbed within this optoelectronic device architecture when the spectral match between the emissive LIL and the absorbing BHJ layer is large.

\section{Acknowledgements}

Richard Pfeifer is acknowledged for his support with the optical simulations and related discussions.

\section{Notes and references}

1 R. Koeppe, O. Boassart, G. Galzaferri and N. S. Sariciftci, Sol. Energy Mater. Sol. Cells, 2007, 91, 155.

2 S. Iijima, F. Mizutani and Y. Tanaka, Bull. Chem. Soc. Jpn., 1985, 58, 1585.

3 E. A. Katz, D. Faiman, S. M. Tuladhar, J. M. Kroon, M. Wienk, T. Fromherz, F. Padinger, C. J. Brabec and N. S. Sariciftci, J. Appl. Phys., 2001, 90, 5343. 
4 I. Riedel, J. Parisi, V. Dyakonov, L. Lutsen, D. Vaderzande and J. C. Hummelen, Adv. Funct. Mater., 2004, 14, 38.

5 A. S. Riad, S. M. Khalil and S. Darwish, Thin Solid Films, 1994, 249, 219.

6 J.-S. Huang, T. Goh, X. Li, M. y. Sfeir, E. A. Bielinski, S. Tomasulo, M. L. Lee, N. Hazari and A. D. Taylor, Nat. Photonics, 2013, 7, 479.

7 T. Ameri, P. Khoram, J. Min and C. J. Brabec, Adv. Mater., 2013, 25, 4245.

8 B. Maennig, D. Gebeyehu, P. Simon, F. Kozlowski, A. Werner, F. Li, S. Grundmann, S. Sonntag, M. Koch, K. Leo, M. Pfeiffer, H. Hoppe, D. Meissner, N. Sariciftci, I. Riedel, V. Dyakonov, J. Parisi and J. Drechsel, Appl. Phys. A: Mater. Sci. Process., 2004, 79, 1.
9 V. Bulovic, A. Shoustikov, M. A. Baldo, E. Bose, V. G. Kozlov, M. e. Thompson and S. R. Forrest, Chem. Phys. Lett., 1998, 287, 455.

10 J. Meiss, M. Furno, S. Pfuetzner, K. Leo and M. Riede, J. Appl. Phys., 2010, 107, 053117.

11 M. Hermenau, M. Riede and K. Leo, Stability and Degradation of Organic and Polymer Solar Cells, ed. F. C. Krebs, Wiley-VCH, 2012.

12 H.-W. Lin, S.-W. Chiu, L.-Y. Lin, Z.-Y. Hung, Y.-H. Chen, F. Lin and K.-T. Wong, Adv. Mater., 2012, 24, 2269.

13 H.-W. Chong, J. Lee, S. Hofmann, Y. H. Kim, L. MüllerMeskamp, B. Lüssem, C.-C. Wu, K. Leo and M. C. Gather, J. Appl. Phys., 2013, 113, 204502. 